\title{
ATRIBUTOS FÍSICOS DO SOLO EM ÁREA DE MINERAÇÃO DE CARVÃO INFLUENCIADOS PELA CORREÇÃO DA ACIDEZ, ADUBAÇÃO ORGÂNICA E REVEGETAÇÃO ${ }^{(1)}$
}

\author{
Antonio Lunardi Neto ${ }^{(2)}$, Jackson Adriano Albuquerque ${ }^{(3)}$, Jaime Antonio de Almeida ${ }^{(3)}$, \\ Álvaro Luiz Mafra ${ }^{(3)}$, João Carlos Medeiros ${ }^{(2)} \&$ Adálcio Alberton $^{(4)}$
}

\section{RESUMO}

Solos reconstruídos após mineração de carvão têm suas propriedades físicas, químicas e biológicas alteradas. Nesse sentido, este trabalho foi desenvolvido com o objetivo de avaliar efeitos da aplicação de corretivos, da adubação e da revegetação nas propriedades físicas de um solo reconstruído após mineração a céu aberto. $O$ experimento foi conduzido na Mina do Apertado, em Lauro Müller, SC, entre 2001 e 2005, sobre um solo minerado em 1991 e reconstruído em 1995. O delineamento experimental foi de blocos ao acaso, com três repetições. Os tratamentos foram: (1) testemunha; (2) “dregs" (resíduo alcalino); (3) calcário; (4) calcário + Brachiaria brizantha; (5) calcário + Brachiaria brizantha + cama de aviário. Em todos os tratamentos, foram transplantadas mudas de Pinus taeda e de Eucalyptus saligna em subparcelas. O calcário dolomítico e o "dregs" foram incorporados a $10 \mathrm{~cm}$ de profundidade. Toda a área recebeu adubação nitrogenada, potássica e fosfatada conforme recomendação técnica. As amostras de solo foram coletadas em fevereiro de 2005, nas camadas de 0 a 5 e $510 \mathrm{~cm}$, avaliando-se os seus atributos físicos. Os tratamentos não alteraram a densidade de partículas, densidade do solo, porosidade total, macro e microporosidade do solo. O calcário elevou o pH, dispersou a argila e reduziu a estabilidade de agregados, enquanto o "dregs" aumentou o pH sem dispersar a argila. A adubação orgânica e o uso de Brachiaria brizantha aumentaram a estabilidade dos agregados e a água prontamente disponível, favorecendo a recuperação da qualidade física do solo degradado.

Termos de indexação: “dregs", recuperação de solos, estrutura do solo.

\footnotetext{
(1) Parte da Dissertação do primeiro autor, apresentada à Universidade do Estado de Santa Catarina - UDESC. Recebido para publicação em janeiro de 2007 e aprovado em maio de 2008.

(2) Mestre em Ciência do Solo pela Universidade do Estado de Santa Catarina - UDESC. Av. Luiz de Camões 2090, CEP 88520-000 Lages (SC). Bolsista da Capes. E-mails: a8aln@cav.udesc.br; medeiros.jc@gmail.com

(3) Professor do Departamento de Solos, CAV/UDESC. E-mails: albuquerque@pq.cnpq.br; a2jaa@cav.udesc.br; a2alm@cav.udesc.br

(4) Estudante da Agronomia, CAV/UDESC. E-mail: adalcio.a@gmail.com
} 


\title{
SUMMARY: CHANGES IN SOIL PHYSICAL PROPERTIES OF A COAL MINING SITE INFLUENCED BY LIMING, POULTRY MANURE AND REVEGETATION
}

\begin{abstract}
The physical, chemical and biological properties of soils of reconstructed areas after coal mining are altered. This study aimed to evaluate liming, mineral and organic fertilizers and revegetation effects on the physical properties of a reconstructed soil after open-pit mining. The experiment was carried out in a former mine in Lauro Müller County - SC, in southern Brazil, from 2001 to 2005. The site was mined in 1991 and reconstructed in 1995. A randomized complete block design was used, with three replications. The treatments consisted of: (1) Control; (2) "dregs" (alkaline residue); (3) Lime; (4) Lime + Brachiaria brizantha; (5) Lime + Brachiaria brizantha + poultry litter. Pinus taeda and Eucalyptus saligna seedlings were transplanted to subplots of all treatments. Dolomitic limestone and dregs were incorporated in the soil at a depth of $10 \mathrm{~cm}$. Nitrogen, potassium and phosphorous fertilizers were added on the soil surface of all plots according to the official recommendation. Soil samples were collected in February 2005 (from the layers $0-5$ and $5-10 \mathrm{~cm}$ ), and their physical and chemical properties evaluated. The treatments had no effects on soil bulk density, particle density, total porosity, macroporosity and microporosity. Liming increased $\mathrm{pH}$, caused clay dispersion and reduced aggregates stability in water, while dregs increased $\mathrm{pH}$ without dispersing clay. Poultry litter and Brachiaria brizantha increased the aggregate stability and volumetric water content, thus favoring the recovery of soil physical quality in degraded areas.
\end{abstract}

Index terms: dregs, soil recovery, soil structure.

\section{INTRODUÇÃO}

A mineração de carvão a céu aberto é uma das principais formas de degradação ambiental na região sul de Santa Catarina, conhecida como região carbonífera. Nessa atividade, é necessário remover, em escavações, solo, subsolo e materiais subjacentes, compostos por rochas e, ou, materiais estéreis que se encontram sobre o carvão, sendo então depositados em locais vizinhos daquele da extração do carvão até o término da exploração da mina, para finalmente serem recolocados no lugar original por maquinários pesados.

Apesar de importante fonte energética, a exploração de carvão é responsável pelo acúmulo desordenado de grande volume de rejeitos, pois efetua-se apenas o nivelamento das pilhas, com recomposição topográfica das áreas utilizadas para depósito, e cobre-se a superfície com uma camada de solo (Gaivizzo et al., 2002). Nesse processo, geralmente não há maiores cuidados com o estado original do solo e subsolo, ocorrendo mistura de horizontes empilhados juntamente com materiais estéreis (Bradshaw, 1997).

Para reduzir esses problemas na recomposição do solo, as camadas e horizontes devem ser separados durante as escavações, para, posteriormente, serem colocados conforme a ordem original em que se apresentavam, atenuando os efeitos indesejáveis oriundos de sua retirada. Entretanto, em muitas minas, isso não é feito adequadamente por questões de custos, originando solos reconstruídos com características indesejáveis, como acidez elevada, desestruturação física e baixa atividade biológica. A principal reação química responsável pela contaminação do solo é a oxidação da pirita $\left(\mathrm{FeS}_{2}\right) \mathrm{em}$ presença de água e $\mathrm{O}_{2}$, que tem alto poder de acidificação da área reconstruída, solubilizando alguns elementos tóxicos para as plantas, sendo necessárias elevadas quantidades de corretivos para neutralizar a acidez do solo (Soares et al., 2006).

Além da alteração química, os solos reconstruídos podem apresentar alterações físicas durante as etapas de remoção e recomposição dos materiais empilhados (McSweeney \& Janzen, 1984). Nos locais minerados, a vegetação original é inevitavelmente destruída, e os solos são perdidos ou enterrados com os resíduos, alterando suas condições estruturais e hídricas (Shukla et al., 2004). Nessas condições, a restrição de ordem física mais comum é a redução da macroporosidade e da infiltração de água, que ocasiona aumento do escoamento superficial e do transporte de solo pela erosão, comprometendo o processo de recuperação das áreas degradadas (Guebert \& Gardner, 2001). Esses autores observaram que, logo após a mineração e recomposição do solo, a taxa de infiltração de água variou entre 1 e $2 \mathrm{~cm} \mathrm{~h}^{-1}$, e, após quatro anos da recomposição, aumentou para $8 \mathrm{~cm} \mathrm{~h}^{-1}$, com valor próximo da infiltração das áreas nãomineradas. Kämpf et al. (1997) observaram alta densidade, com variação de 1,41 a $2,03 \mathrm{Mg} \mathrm{m}^{-3}$, em solo reconstruído após a mineração do carvão no Rio Grande do Sul. Esse grau de compactação tornou esse solo menos permeável à água e mais suscetível ao escoamento superficial e à erosão hídrica. 
Segundo Melloni et al. (2003), a recuperação dos fungos micorrízicos arbusculares em área impactada pela mineração é mais relacionada com a vegetação introduzida do que com o tempo de reabilitação das áreas. Assim, o manejo adequado do solo e das culturas pode reduzir o tempo necessário para recuperação de áreas degradadas. A calagem, a fertilização e o plantio de espécies forrageiras e arbóreas são práticas necessárias em áreas mineradas, conforme observado por Maçaneiro (2001) e Moreira (2004).

As indústrias de celulose, por sua vez, geram resíduos alcalinos como o "dregs" (Albuquerque et al., 2002), que podem ser testados para aplicação nas áreas reconstruídas que necessitam de correção da acidez, evitando sua deposição em aterros. Em estudo realizado por Jordan \& Rodriguez (2004), com a adição de "dregs" em plantio das espécies Pinus radiata e Eucalyptus globulus, foi observado o aumento de crescimento das plantas. A adição de dois resíduos alcalinos da indústria de papel e celulose melhorou as propriedades físicas e químicas de solos degradados (Zambrano et al., 2003). Salienta-se que o descarte de "dregs" em solos deve ser feito com base em critérios técnicos, especialmente pela presença de $\mathrm{Na}$ em sua constituição, que, associado à elevação do $\mathrm{pH}$, pode dispersar a argila (Waldemar \& Herrera, 1986). Após análise da composição química inorgânica do "dregs", Almeida et al. (2007) concluíram que este resíduo pode ser utilizado como corretivo da acidez e como fonte de Ca em solos agrícolas sem risco de contaminação.

A área denominada Mina do Apertado, em Lauro Müller (SC), vem servindo de base experimental para estudos científicos visando à recuperação de solos degradados pela mineração de carvão a céu aberto. Nessa área, foi detectada deficiência na reconstrução do solo pela disposição inadequada de materiais e resíduos da mineração, com prejuízo às propriedades físicas e químicas do solo (Campos et al., 2003), sendo necessário corrigir tais deficiências para permitir sua revegetação. Este trabalho foi desenvolvido com o objetivo de avaliar os efeitos da correção da acidez, da adubação orgânica e da revegetação sobre as propriedades físicas do solo reconstruído em área de mineração de carvão a céu aberto.

\section{MATERIAL E MÉTODOS}

O experimento foi implantado na Mina do Apertado, localizada no Distrito de Guatá, em Lauro Müller (SC) (coordenadas $28^{\circ} 20^{\prime} \mathrm{S}$ e $49^{\circ} 20^{\prime} \mathrm{W}$ ). O local foi minerado em 1991 e o solo foi reconstruído em 1995.

Segundo a classificação de Köppen, o clima é do tipo mesotérmico úmido com verão quente (Cfa), com precipitação pluvial e temperatura média anual de $1.400 \mathrm{~mm} \mathrm{e} 19^{\circ} \mathrm{C}$ (Santa Catarina, 1991). A geologia local é constituída por rochas sedimentares das formações gonduânicas da bacia do Paraná, representadas localmente pelas formações Palermo e Rio Bonito, Grupo Guatá, especificamente a camada minerada Barro Branco (Brasil, 1987). Campos et al. (2003) analisaram um solo adjacente à área minerada e classificaram-no como Argissolo Vermelho-Amarelo alítico típico (Embrapa, 2006).

Em 2001, foi iniciado o manejo do solo reconstruído para a implantação do experimento, com escarificação, remoção manual de rochas para fora da área e incorporação parcial de calcário com grade niveladora. $\mathrm{O}$ delineamento experimental consistiu de blocos ao acaso, com três repetições, sendo as parcelas com dimensões de 12,5 x 9 m. Em novembro de 2001, foram aplicados os seguintes tratamentos: (1) testemunha (Test); (2) calcário (Calc); (3) calcário + Brachiaria brizantha (CalcBb); e (4) calcário + Brachiaria brizantha + cama de aviário (CalcBbCa).

A composição química da cama de aviário não foi analisada, sendo considerados os teores de nutrientes médios segundo a Comissão de Química e Fertilidade do Solo (CQFSRS/SC, 2004). Com os objetivos de favorecer o processo de revegetação e aumentar o teor de matéria orgânica do solo, foi aplicada cama de aviário em dose única ( $6 \mathrm{Mg} \mathrm{ha}^{-1} \mathrm{em}$ base seca). Esta dose supre aproximadamente a metade do requerimento de $\mathrm{N}$ pela Brachiaria e adiciona $\mathrm{P}$ e $\mathrm{K}$ acima da quantidade recomendada (CQFSRS/SC, 2004).

A dose de calcário foi de $25 \mathrm{Mg} \mathrm{ha}^{-1}$, com PRNT $100 \%$, visando elevar o $\mathrm{pH}$ do solo para 6,0. Na instalação do experimento, em toda a área, o solo foi suplementado com $66 \mathrm{~kg} \mathrm{ha}^{-1}$ de $\mathrm{N}$ na forma de uréia, $110 \mathrm{~kg} \mathrm{ha}^{-1} \mathrm{de}_{2} \mathrm{O}_{5}$ na forma de superfosfato triplo e $110 \mathrm{~kg} \mathrm{ha}^{-1} \mathrm{de}_{2} \mathrm{O}$ na forma de cloreto de potássio, de acordo com a recomendação para gramíneas forrageiras de estação quente (CQFSRS/SC, 2004).

A gramínea foi semeada a lanço, com $6,0 \mathrm{~kg} \mathrm{ha}^{-1}$ de sementes. A espécie arbórea utilizada na revegetação, nesta etapa, foi o eucalipto (Eucalyptus saligna), transplantado em toda a área do experimento, num espaçamento de 1,5 x 2,5 m, sendo adicionados mais $40 \mathrm{~g}$ de calcário, por cova, em todos os tratamentos.

Apesar dos tratamentos e da adição da adubação mineral, o eucalipto não apresentou crescimento adequado, devido às restrições químicas e físicas do solo, sendo arrancadas suas mudas em agosto de 2004. Além disso, a Brachiaria apresentava crescimento abaixo da capacidade de produção comparada com a de solo agrícola. A principal restrição física era o baixo volume de macroporos $\left(0,07 \mathrm{~m}^{3} \mathrm{~m}^{-3}\right)$ em relação aos microporos $\left(0,41 \mathrm{~m}^{3} \mathrm{~m}^{-3}\right)$. Além disso, devido à quantidade elevada de pirita na camada superficial e sua oxidação e ao conseqüente abaixamento do $\mathrm{pH}$, houve necessidade de realizar nova correção da acidez. Havia sintoma de deficiência de $\mathrm{P}$ nas folhas do eucalipto. 
Nessa época, houve então a reformulação do experimento, sendo os quatro tratamentos originais reaplicados nas respectivas parcelas.

A acidez do solo foi novamente corrigida, aplicandose $13 \mathrm{Mg} \mathrm{ha}^{-1}$ de calcário com PRNT $100 \%$, distribuídos manualmente e incorporados com grade aradora. A dose foi calculada para a camada de 0 a $20 \mathrm{~cm}$ conforme recomendação (CQFSRS/SC, 2004); no entanto, devido à presença de rochas, a incorporação não foi realizada adequadamente.

A cama de aviário foi aplicada na dose de $9 \mathrm{Mg}_{\mathrm{ga}}{ }^{-1}$ em base seca, para estimular o crescimento e desenvolvimento inicial das árvores. A dose de cama de aviário foi maior do que a aplicada em 2001, pois foi observada deficiência de $\mathrm{N}$ nas culturas, devido ao baixo teor de carbono (C) contido no material utilizado para recuperar a área e à imobilização do $\mathrm{N}$ pelos microrganismos, quando da decomposição dos resíduos vegetais da Brachiaria, que era roçada e deixada sobre o solo como cobertura.

Nessa ocasião, introduziu-se um tratamento adicional com "dregs", um resíduo alcalino da indústria de celulose (Quadro 1). A dose foi correspondente ao somatório das doses de calcário que foram aplicadas nos tratamentos Calc, $\mathrm{CalcBb}$ e CalcBbCa, efetuandose a correção de acordo com seu poder corretivo de neutralização.

Assim como na implantação do experimento, o solo foi adubado com $66 \mathrm{~kg} \mathrm{ha}^{-1}$ de $\mathrm{N}$ na forma de uréia,

\section{Quadro 1. Análise química do resíduo alcalino da indústria de papel e celulose ("dregs")}

\section{Elemento (Unidade) Teor Limite de detecção}

\begin{tabular}{|c|c|c|}
\hline $\mathrm{pH}$ & 10,9 & \\
\hline Nitrogênio (NKT) $(\%)^{(1)}$ & 0,02 & $0,01 \%$ \\
\hline Fósforo (\%) & 0,08 & $0,01 \%$ \\
\hline Potássio (\%) & 0,23 & $0,01 \%$ \\
\hline Cálcio (\%) & 30,0 & $0,01 \%$ \\
\hline Magnésio (\%) & 1,0 & $0,01 \%$ \\
\hline Enxofre (\%) & 0,71 & $0,01 \%$ \\
\hline Cobre $\left(\mathrm{mg} \mathrm{kg}^{-1}\right)$ & 61,0 & $0,6 \mathrm{mg} \mathrm{kg}^{-1}$ \\
\hline Zinco (mg kg-1 $)$ & 189 & $2 \mathrm{mg} \mathrm{kg}^{-1}$ \\
\hline Ferro $(\%)$ & 0,29 & $4 \mathrm{mg} \mathrm{kg}^{-1}$ \\
\hline Manganês (\%) & 0,40 & $4 \mathrm{mg} \mathrm{kg}^{-1}$ \\
\hline Sódio (\%) & 3,4 & $0,01 \%$ \\
\hline Cádmio (mg kg $\left.{ }^{-1}\right)$ & 0,9 & $0,2 \mathrm{mg} \mathrm{kg}^{-1}$ \\
\hline Cromo $\left(\mathrm{mg} \mathrm{kg}^{-1}\right)$ & 284 & $0,4 \mathrm{mg} \mathrm{kg}^{-1}$ \\
\hline Níquel (mg kg $\left.{ }^{-1}\right)$ & 99 & $0,4 \mathrm{mg} \mathrm{kg}^{-1}$ \\
\hline Chumbo (mg kg$\left.{ }^{-1}\right)$ & 3,0 & $2 \mathrm{mg} \mathrm{kg}^{-1}$ \\
\hline Boro $\left(\mathrm{mg} \mathrm{kg}^{-1}\right)$ & 6,0 & $1 \mathrm{mg} \mathrm{kg}^{-1}$ \\
\hline
\end{tabular}

(1) NKT: nitrogênio de Kjeldahl total. Análise realizada no laboratório de análise de solos da Universidade Federal do Rio Grande do Sul (UFRGS).
$110 \mathrm{~kg} \mathrm{ha}{ }^{-1}$ de $\mathrm{P}_{2} \mathrm{O}_{5}$ na forma de superfosfato triplo e $110 \mathrm{~kg} \mathrm{ha}^{-1}$ de $\mathrm{K}_{2} \mathrm{O}$ na forma de cloreto de potássio (CQFSRS/SC, 2004). Após a correção da acidez e da adubação, a Brachiaria brizantha foi semeada a lanço, com $6,0 \mathrm{~kg} \mathrm{ha}^{-1}$ de sementes.

Em outubro de 2004, na metade de cada parcela foram transplantadas mudas de Pinus taeda e, na outra metade, Eucalyptus saligna em todos os tratamentos, em covas de 0,2 x 0,2 × 0,2 m, espaçadas de 1,5 m entre plantas e 2,5 m entre fileiras. Em cada cova, em todos os tratamentos, foram adicionados $44 \mathrm{~g}$ de $\mathrm{P}_{2} \mathrm{O}_{5}$ e $\mathrm{K}_{2} \mathrm{O}$ e $20 \mathrm{~g}$ de $\mathrm{N}$. Nos tratamentos com calcário e no testemunha, foram adicionados, por cova, 72 g de calcário, e nas parcelas do tratamento com "dregs" foram aplicados 108 g do produto na cova. Em todas as covas, houve a homogeneização do adubo e do corretivo com o solo.

Em dezembro de 2004, foram feitas capinas de coroamento ao redor das mudas transplantadas e roçagem da parte aérea da gramínea, devido ao seu intenso crescimento inicial, permanecendo sobre o solo a massa vegetal cortada. Na mesma data, foram aplicados $40 \mathrm{~kg} \mathrm{ha}^{-1}$ de $\mathrm{N}$ na forma de uréia, a lanço, sem incorporação.

Amostras de solo com estrutura indeformada foram coletadas em fevereiro de 2005 nas camadas de 0 a 5 e 5 a $10 \mathrm{~cm}$, em anéis com $50 \mathrm{~mm}$ de altura e volume de $100 \mathrm{~cm}^{3}$. Os anéis foram acondicionados em latas de $\mathrm{Al}$, lacrados com fita adesiva e levados ao laboratório para a determinação, por ocasião da coleta, da umidade gravimétrica (UG), da água prontamente disponível (APD), da porosidade total (PT), da macro (Macro) e microporosidade (Micro) e da densidade do solo (Ds). Foi coletado o solo com estrutura deformada em leivas para determinação da estabilidade de agregados em água, densidade de partículas (Dp), teores de areia, silte, argila total e argila dispersa em água (AD), para o cálculo do grau de floculação (GF) e para caracterização química em termos de acidez e disponibilidade de nutrientes (Tedesco et al., 1995).

As amostras em anéis volumétricos foram saturadas e submetidas, seqüencialmente, à tensão de $6 \mathrm{kPa}$ em mesa de tensão para determinação de porosidade total, macro e microporosidade, com posterior equilíbrio nas tensões de 10, 33, 50 e $100 \mathrm{kPa}$, em câmara de Richards. A água prontamente disponível (APD) foi calculada pela diferença entre a água retida entre as tensões de 10 e $100 \mathrm{kPa}$ (Costa, 2005). As amostras foram secas em estufa para determinar a massa de solo seco, obtendo-se a densidade do solo.

Para a determinação da estabilidade de agregados em água, foram utilizados agregados entre 8,0 e 4,76 mm de diâmetro, sendo os resultados expressos pelo diâmetro médio ponderado (DMP, em $\mathrm{mm}$ ). As peneiras utilizadas para agitação em água foram de 4,76, 2,00, 1,00 e 0,25 mm. Antes da agitação, os agregados secos ao ar foram umedecidos por capilaridade, por $10 \mathrm{~min}$, 
e depois foram submetidos à agitação também por 10 min, com 40 oscilações min $^{-1}$ e curso de oscilação vertical de $3,8 \mathrm{~cm}$, segundo o método de Kemper \& Chepil (1965). Os agregados menores que $0,25 \mathrm{~mm}$ foram denominados microagregados.

Nas amostras coletadas com estrutura deformada, determinou-se a distribuição granulométrica pelo método da pipeta. Em frascos de vidro de $150 \mathrm{~mL}$, foram adicionados solo, água e $\mathrm{NaOH}$ e duas bolas de acrílico. Após repouso por uma noite, foram agitados com 150 ciclos min $^{-1}$ durante duas horas (Suzuki et al., 2004). Na determinação da argila dispersa, utilizou-se apenas a água como dispersante, com a mesma agitação. A densidade de partículas foi analisada pelo método do balão volumétrico (Embrapa, 1997).

A análise estatística consistiu da análise de normalidade dos dados por meio do método de ShapiroWilk, seguido da análise da variância, para o modelo experimental de blocos ao acaso com parcelas subdivididas, com os tratamentos nas parcelas principais e as camadas nas subparcelas. A comparação entre médias de tratamentos foi avaliada pelo teste DMS (5 \%). Utilizou-se o teste de correlação de Pearson para verificar relações entre os atributos avaliados.

\section{RESULTADOS E DISCUSSÃO}

Os efeitos dos tratamentos foram significativos para DMP, microagregados, AD e GF. Houve efeito significativo da interação entre tratamento e camada para $\mathrm{AD}, \mathrm{GF}$ e $\mathrm{APD}$. Os demais atributos físicos não foram modificados significativamente pelos tratamentos ou camadas (Quadro 2).

O solo reconstruído apresentou, em média, $350 \mathrm{~g} \mathrm{~kg}^{-1}$ de areia, 370 de silte e 280 de argila na camada de 0 a $10 \mathrm{~cm}$; na testemunha e no tratamento com "dregs", a argila dispersa foi próxima de zero; nos tratamentos com calcário, a dispersão das partículas aumentou, especialmente na camada de 0 a $5 \mathrm{~cm}$, com $135 \mathrm{~g} \mathrm{~kg}^{-1}$ no tratamento $\mathrm{CalcBb}$ e $222 \mathrm{~g} \mathrm{~kg}^{-1}$ no tratamento CalcBbCa (Figura 1a). Isso ocorreu pelo aumento do $\mathrm{pH}$ para 7,0 e 7,3, respectivamente (Quadro 3), e conseqüente criação de cargas negativas dependentes do $\mathrm{pH}$, o que pode levar à repulsão das cargas e à dispersão do solo (Albuquerque et al., 2000). Esta hipótese é reforçada pela correlação positiva entre $\mathrm{pH}$ em água e argila dispersa $(r=0,59 * *$; Quadro 4).

Uma diferença importante entre o calcário e o "dregs" é a composição dos cátions, sendo o "dregs" composto principalmente por Ca e em menor teor por $\mathrm{Na}$ e $\mathrm{Mg}$ (Quadro 1), e o calcário, por $\mathrm{Ca}$ e $\mathrm{Mg}$. Waldemar \& Herrera (1986) encontraram no "dregs" $35 \%$ de $\mathrm{CaO}, 3,6 \%$ de $\mathrm{MgO}$ e 4,7 \% de $\mathrm{Na}$ em base seca. No tratamento com "dregs", devido à presença de $\mathrm{Na}$ (Quadro 3), esperava-se maior dispersão da argila, o que não foi observado pelo aumento na concentração iônica da solução, devido ao aumento de sais em virtude da maior solubilidade do "dregs" em relação ao calcário. O maior teor de sais comprime a dupla camada elétrica dos colóides e mantém a floculação. Além disso, o $\mathrm{Na}$ é um íon retido fracamente nas partículas, sendo facilmente deslocado, por ser monovalente, por cátions de maior valência quando em iguais teores. Se deslocado, vai para a solução e pode ser absorvido ou então lixiviado (Agassi et al., 1981; Miller et al., 1990). Observou-se também que o $\mathrm{pH}$ do solo tratado com "dregs" ficou em 6,6 na camada de 0 a $5 \mathrm{~cm}$, inferior aos tratamentos com calcário (Quadro 3). Isso pode indicar um valor de $\mathrm{pH}$ acima do qual a dispersão é favorecida. Esse valor de $\mathrm{pH}$ pode estar entre 6,6 do tratamento com "dregs" e 7,3 com calcário.

Nos tratamentos em que o teor de argila dispersa foi menor (Figura 1a), o grau de floculação aumentou (Figura 1b). No solo reconstruído, com elevada acidez, o grau de floculação do testemunha foi próximo de $100 \%$, pois o $\mathrm{pH}$ está próximo do ponto de carga zero e, assim, a floculação das partículas é favorecida. A adição de calcário reduziu sensivelmente o grau de floculação, principalmente na camada de 0 a $5 \mathrm{~cm}$.

Quadro 2. Resumo da análise de variância para as causas de variação: tratamento, camada e a interação tratamento x camada para alguns atributos físicos do solo da área reconstruída

\begin{tabular}{lccccccccccccc}
\hline Causa de variação & $\mathbf{A D}^{(1)}$ & GF & Dp & Ds & Ma & Mi & PT & APD & UV & MAg & DMP \\
\hline Tratamento & $* *(2)$ & $* *$ & $\mathrm{~ns}$ & $\mathrm{~ns}$ & $\mathrm{~ns}$ & $\mathrm{~ns}$ & $\mathrm{~ns}$ & $\mathrm{~ns}$ & $\mathrm{~ns}$ & $* *$ & $*$ \\
Camada & $* *$ & $* *$ & $\mathrm{~ns}$ & $\mathrm{~ns}$ & $\mathrm{~ns}$ & $\mathrm{~ns}$ & $\mathrm{~ns}$ & $\mathrm{~ns}$ & $\mathrm{~ns}$ & $\mathrm{~ns}$ & $\mathrm{~ns}$ \\
Trat x Camada & $* *$ & $*$ & $\mathrm{~ns}$ & $\mathrm{~ns}$ & $\mathrm{~ns}$ & $\mathrm{~ns}$ & $\mathrm{~ns}$ & $* *$ & $\mathrm{~ns}$ & $\mathrm{~ns}$ & $\mathrm{~ns}$ \\
\hline
\end{tabular}

(1) AD: argila dispersa em água; GF: grau de floculação; Dp: densidade de partículas; Ds: densidade do solo; Ma: macroporosidade; Mi: microporosidade; PT: porosidade total; APD: água prontamente disponível; UV: umidade volumétrica; MAg: percentagem de microagregados menores do que $0,25 \mathrm{~mm}$; e DMP: diâmetro médio ponderado. ${ }^{(2)}$ ns: não-significativo; ** $\mathrm{e}$ * significativo a $1 \mathrm{e}$ $5 \%$, respectivamente, pelo teste $\mathrm{F}$. 
Quadro 3. Valores de pH, teores de sódio, densidade de partículas (Dp), densidade do solo (Ds), macroporosidade (Ma), microporosidade (Mi), porosidade total (PT) e umidade volumétrica do solo (UV) nos diferentes tratamentos, em coleta de solo realizada em fevereiro de 2005

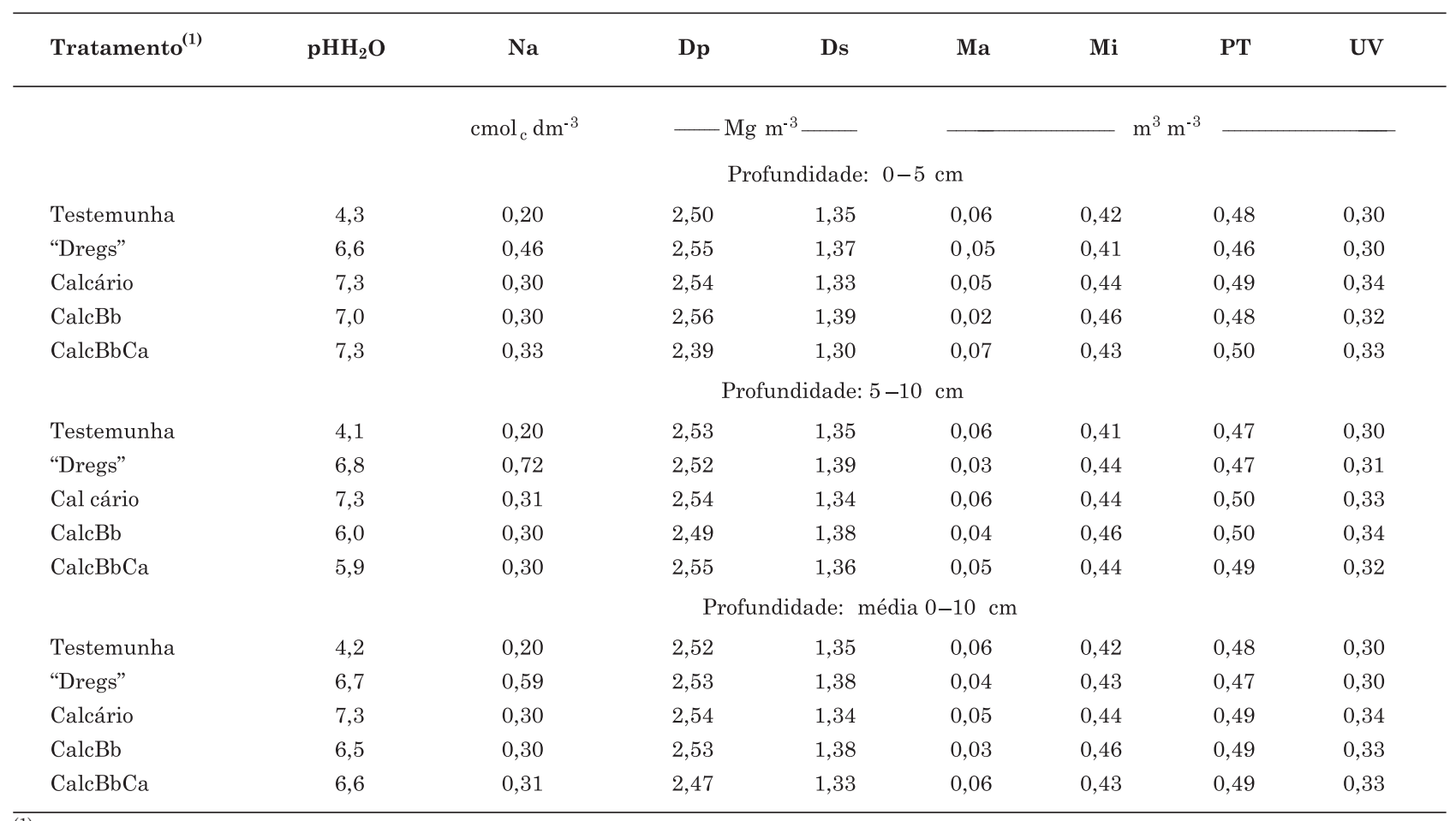

(1) CalcBb: calcário + Brachiaria brizantha; CalcBbCa: calcário + Brachiaria brizantha + cama de aviário.

Quadro 4. Correlações de Pearson entre as propriedades físicas e químicas do solo reconstruído após exploração de carvão, considerando todos os tratamentos de recuperação e camadas

\begin{tabular}{|c|c|c|c|c|c|c|c|c|c|}
\hline & $\mathrm{AD}^{(1)}$ & GF & Ds & Ma & Mi & PT & APD & MAg & DMP \\
\hline $\mathrm{pHH}_{2} \mathrm{O}$ & $0,59 * *(2)$ & $-0,59^{* *}$ & $\mathrm{~ns}$ & $\mathrm{~ns}$ & $0,29^{*}$ & $\mathrm{~ns}$ & ns & $\mathrm{ns}$ & $\mathrm{ns}$ \\
\hline $\mathrm{K}$ & $0,40 * *$ & $-0,37^{* *}$ & ns & ns & ns & ns & $\mathrm{ns}$ & $\mathrm{ns}$ & ns \\
\hline $\mathrm{Na}$ & ns & $\mathrm{ns}$ & ns & ns & ns & ns & $0,28^{*}$ & $0,31^{*}$ & $-0,26^{*}$ \\
\hline $\mathrm{Ca}$ & $\mathrm{ns}$ & $\mathrm{ns}$ & $\mathrm{ns}$ & $\mathrm{ns}$ & $\mathrm{ns}$ & $\mathrm{ns}$ & $0,28^{*}$ & $\mathrm{~ns}$ & $\mathrm{~ns}$ \\
\hline $\mathrm{Mg}$ & $0,49 * *$ & $-0,47^{* *}$ & $\mathrm{~ns}$ & $\mathrm{~ns}$ & $0,27^{*}$ & $0,29^{*}$ & $\mathrm{~ns}$ & $\mathrm{~ns}$ & $\mathrm{~ns}$ \\
\hline MO & ns & $\mathrm{ns}$ & $\mathrm{ns}$ & $\mathrm{ns}$ & $\mathrm{ns}$ & $\mathrm{ns}$ & $\mathrm{ns}$ & $-0,30^{*}$ & $0,34^{*}$ \\
\hline SB & $\mathrm{ns}$ & $\mathrm{ns}$ & $\mathrm{ns}$ & $\mathrm{ns}$ & ns & $\mathrm{ns}$ & $0,29^{*}$ & ns & $\mathrm{ns}$ \\
\hline CTC pH7 & $\mathrm{ns}$ & $\mathrm{ns}$ & ns & ns & ns & ns & 0,30 * & $\mathrm{ns}$ & ns \\
\hline $\mathrm{V}$ & $0,28^{*}$ & $-0,28^{*}$ & $\mathrm{~ns}$ & $-0,25^{*}$ & ns & $\mathrm{ns}$ & $0,26^{*}$ & $\mathrm{~ns}$ & ns \\
\hline M & $-0,38^{* *}$ & $0,38^{* *}$ & $\mathrm{~ns}$ & $\mathrm{~ns}$ & $-0,26^{*}$ & $\mathrm{~ns}$ & $-0,27^{*}$ & $\mathrm{~ns}$ & $\mathrm{~ns}$ \\
\hline $\mathrm{ST}$ & $\mathrm{ns}$ & $\mathrm{ns}$ & ns & $-0,28^{*}$ & $\mathrm{~ns}$ & ns & $\mathrm{ns}$ & $0,36^{* *}$ & $-0,33^{* *}$ \\
\hline Areia & ns & ns & ns & ns & ns & ns & ns & $0,25^{*}$ & $-0,30^{*}$ \\
\hline Silte & $\mathrm{ns}$ & $\mathrm{ns}$ & $\mathrm{ns}$ & $-0,26^{*}$ & $0,32^{* *}$ & $\mathrm{~ns}$ & ns & $\mathrm{ns}$ & $\mathrm{ns}$ \\
\hline Arg & $\mathrm{ns}$ & $\mathrm{ns}$ & $\mathrm{ns}$ & $\mathrm{ns}$ & ns & $\mathrm{ns}$ & ns & $\mathrm{ns}$ & $0,30^{*}$ \\
\hline $\mathrm{AD}$ & & $-0,98^{* *}$ & ns & ns & $0,28^{*}$ & $0,25^{*}$ & ns & $\mathrm{ns}$ & $\mathrm{ns}$ \\
\hline GF & & & ns & $\mathrm{ns}$ & $-0,28^{*}$ & $\mathrm{~ns}$ & $\mathrm{~ns}$ & $\mathrm{~ns}$ & ns \\
\hline Ds & & & & $-0,72^{* *}$ & $\mathrm{~ns}$ & $-0,79^{* *}$ & $-0,37^{* *}$ & $\mathrm{~ns}$ & ns \\
\hline $\mathrm{Ma}$ & & & & & $-0,47^{\star * *}$ & $0,47^{* *}$ & $\mathrm{~ns}$ & $\mathrm{~ns}$ & $\mathrm{~ns}$ \\
\hline $\mathrm{Mi}$ & & & & & & $0,54^{* *}$ & ns & ns & ns \\
\hline $\mathrm{PT}$ & & & & & & & ns & $\mathrm{ns}$ & ns \\
\hline APD & & & & & & & & $\mathrm{ns}$ & ns \\
\hline MAg & & & & & & & & & $\mathrm{ns}$ \\
\hline
\end{tabular}

(1) AD: argila dispersa; GF: grau de floculação; Ds: densidade do solo; Ma: macroporosidade; Mi: microporosidade; PT: porosidade total; APD: água prontamente disponível; MAg: microagregados menores que $0,25 \mathrm{~mm}$; e DMP: diâmetro médio ponderado. ${ }^{(2)} * *$ significativo $(p<0,01)$; * significativo $(p=0,01-0,05)$; ns: não-significativo $(p>0,05)$. 
Essa redução é especialmente danosa em solos degradados, pois a argila dispersa, ao obstruir poros, reduz a taxa de infiltração e favorece o escoamento superficial. Albuquerque et al. (2000) observaram que a adição de calcário reduziu o grau de floculação de um Latossolo Bruno quando o $\mathrm{pH}$ foi superior a 6,0.

Os tratamentos não alteraram a densidade de partículas, a densidade do solo, a macroporosidade, a microporosidade e a porosidade total (Quadro 3). Para solos de textura franco argilosa, classe textural do solo deste experimento, a densidade crítica do solo situase entre 1,4 e 1,5 $\mathrm{Mg} \mathrm{m}^{-3}$ (Reichert et al., 2003). Neste estudo, a densidade média do solo foi de $1,35 \mathrm{Mg} \mathrm{m}^{-3}$, portanto ligeiramente inferior ao limite crítico proposto por Reichert et al. (2003). Entretanto, pode haver comprometimento dos fluxos hídricos no solo, pois a macroporosidade é inferior a $0,10 \mathrm{~m}^{3} \mathrm{~m}^{-3}$, valor considerado o limite inferior para o bom crescimento e desenvolvimento da maioria das culturas de sequeiro
(Hillel, 1998). Solos com poucos macroporos apresentam sérias restrições ao crescimento vegetal, às trocas gasosas e à infiltração de água, com mais riscos de erosão hídrica, conforme foi constatado visualmente nessa área. Tais restrições físicas e hídricas reduzem a aeração e o crescimento das plantas em áreas mineradas (Indorante et al., 1981), como reportado em detalhe em solos recuperados após mineração de carvão na região central da Pensilvânia, Estados Unidos (Guebert \& Gardner, 2001). Dessa forma, na área em questão, a macroporosidade deve ser aumentada com matéria orgânica e plantas de cobertura adaptadas ao ambiente, como uma das principais estratégias na recuperação desses solos (Bendfeldt et al., 2001).

O tratamento com calcário (Calc) teve maior volume de microagregados (29\%), em relação aos tratamentos testemunha $(17 \%)$, CalcBb $(17 \%)$ e CalcBbCa (9 \%) (Figura 1c), provavelmente pelo
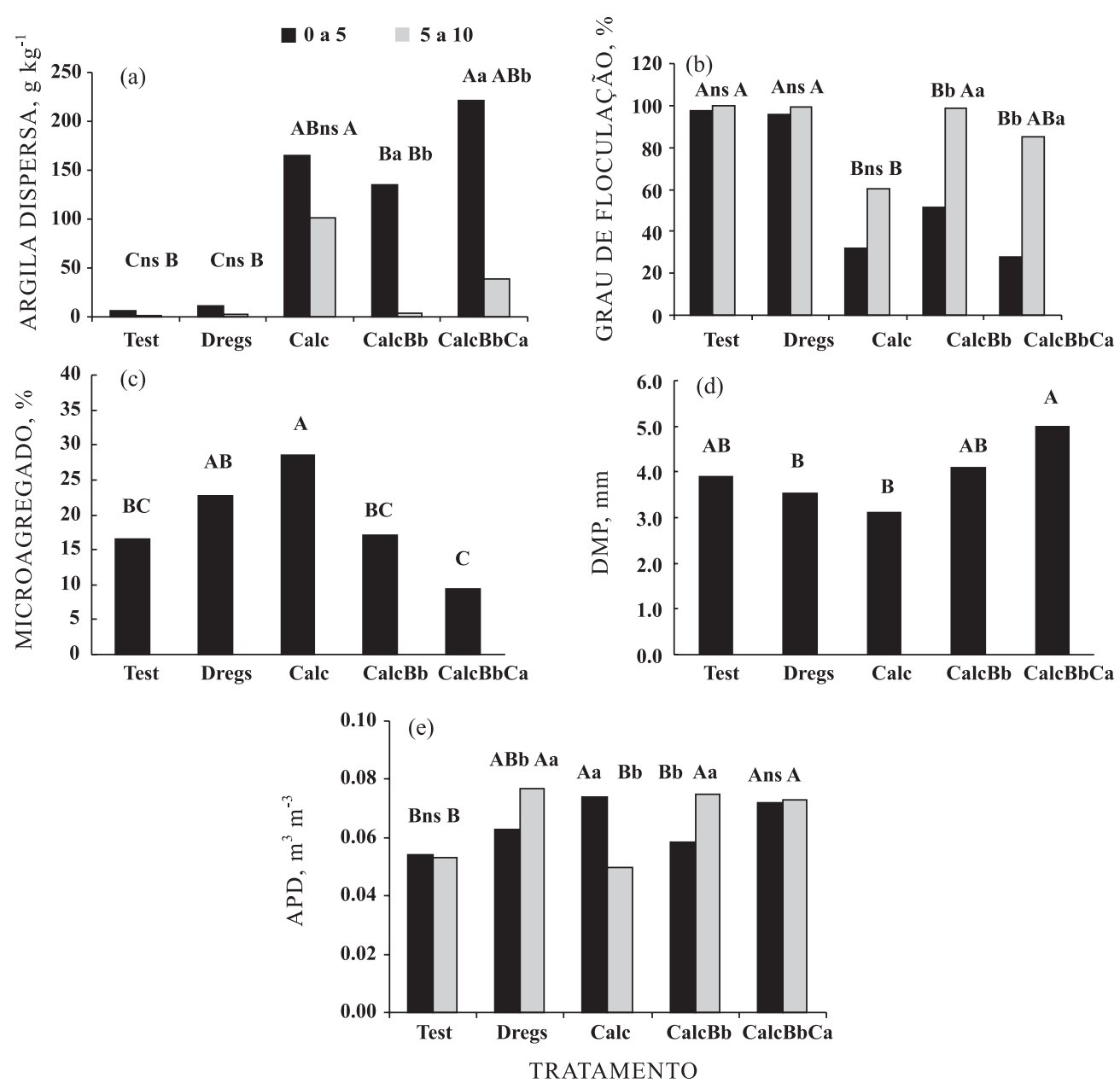

Figura 1. Efeito da interação tratamento x camada na argila dispersa em água (a), grau de floculação (b) e água prontamente disponível (APD) (e) e efeito do tratamento, na média das duas camadas, na percentagem de microagregados (c) e diâmetro médio ponderado (DMP) (d). Letras maiúsculas comparam tratamentos em cada camada (1a, 1b e 1e) ou na média das camadas de 0 a $10 \mathrm{~cm}$ (1c e 1d). Letras minúsculas comparam camadas em cada tratamento (DMS 5 \%). Tes: testemunha; “dregs"; Calc: calcário; CalcBb: calcário + Brachiaria brizantha; CalcBbCa: calcário + Brachiaria brizantha + Cama de aviário. 
aumento do $\mathrm{pH}$ para 7,3, na média da camada de 0 a $10 \mathrm{~cm}$ (Quadro 3), o que favoreceu o enfraquecimento das ligações entre partículas. Com isso, foi observada redução do DMP no tratamento com calcário $(\mathrm{DMP}=$ $3,1 \mathrm{~mm}$ ) em relação ao testemunha $(\mathrm{DMP}=3,9 \mathrm{~mm})$ (Figura 1d). Entretanto, nos tratamentos com calcário + Brachiaria $(\mathrm{DMP}=4,1 \mathrm{~mm})$ e calcário + Brachiaria + cama de aviário $(\mathrm{DMP}=5,0 \mathrm{~mm})$, a estabilidade foi recuperada. $\mathrm{O}$ aumento da estabilidade de agregados pode estar associado ao sistema radicular fasciculado e à deposição dos resíduos vegetais da parte aérea da Brachiaria, conforme observado por Silva \& Mielniczuk (1997) para gramíneas perenes no tratamento $\mathrm{CalcBb}$, pela adição de cama de aviário no tratamento CalcBbCa, os quais promovem a agregação (D'Andréa et al., 2002). Não houve correlação significativa entre estabilidade de agregados e GF (Quadro 4).

A água prontamente disponível (APD), variou de $0,050 \mathrm{~m}^{3} \mathrm{~m}^{-3}$ no tratamento com calcário a $0,077 \mathrm{~m}^{3} \mathrm{~m}^{-3}$ no tratamento com "dregs", ambas na camada de 5 a $10 \mathrm{~cm}$ (Figura 1e). Contudo, nas duas camadas avaliadas, a APD foi menor na testemunha e maior no $\mathrm{CalcBbCa}$, o que pode estar refletindo o efeito da cama de aviário na retenção de água. A compactação e a baixa disponibilidade de água para as plantas são os principais problemas constatados em solos reconstruídos após mineração (Torbert et al., 1990). O aumento da densidade do solo oriundo de reconstrução diminui a infiltração da água e aumenta o escoamento superficial no perfil compactado (Harms \& Chanasyk, 2000). Nesse sentido, além da descompactação mecânica, sugere-se a manutenção permanente de resíduos vegetais na superfície do solo após as operações mecânicas, para reduzir a erosão hídrica durante a recuperação desses solos (Chong \& Cowaert, 1997).

Em estudo realizado em solos reconstruídos após mineração de carvão e em solo em estado natural nãodegradado (Argissolo Vermelho eutrófico típico), Pauletto et al. (2003) concluíram que a quantidade de água disponível entre a capacidade de campo e o ponto de murcha permanente, na camada superficial de $0 \mathrm{a}$ $10 \mathrm{~cm}$, foi baixa e não diferiu entre os diferentes solos. No entanto, nas camadas subsuperficiais, a quantidade de água disponível foi menor no solo reconstruído do que no solo na condição natural.

É possível que o efeito de aplicação de resíduo orgânico, associado com o aporte da parte aérea da gramínea, cortada duas vezes durante o verão, tenha contribuído para o aumento no teor de água disponível no tratamento calcário + Brachiaria + cama de aviário (CalcBbCa). Entretanto, observa-se relação positiva da APD com Na, Ca, soma e saturação por bases e capacidade de troca de cátions e negativa com saturação por $\mathrm{Al}$ e densidade do solo, não havendo relação com distribuição de tamanho das partículas nem com MO (Quadro 4).

\section{CONCLUSÕES}

1. A correção do solo reconstruído após a exploração de carvão deve ser acompanhada da introdução de espécies vegetais que cobrem o solo e pela adição de matéria orgânica para favorecer os mecanismos de floculação da argila e da estabilização dos agregados.

2. O "dregs" elevou o pH e não alterou os teores de argila dispersa e a estabilidade de agregados, sendo seu uso em solos ácidos e reconstruídos uma alternativa viável.

3. A adubação com cama de aviário e a semeadura de Brachiaria brizantha no solo corrigido melhoram as propriedades físicas dos solos reconstruídos.

\section{LITERATURA CITADA}

AGASSI, M.; SHAINBERG, I. \& MORIN, J. Effect of electrolyte concentration and soil sodicity on infiltration rate and crust formation. Soil Sci. Soc. Am. J., 45:848-851, 1981.

ALBUQUERQUE, J.A.; ARGENTON, J.; FONTANA, E.C.; COSTA, F.S. \& RECH, T.D. Propriedades físicas e químicas de solos incubados com resíduo alcalino da indústria de celulose. R. Bras. Ci. Solo, 26:1065-1073, 2002.

ALBUQUERQUE, J.A.; BAYER, C.; ERNANI, P.R. \& FONTANA, E.C. Propriedades físicas e eletroquímicas de um Latossolo Bruno afetadas pela calagem. R. Bras. Ci. Solo, 24:295-300, 2000.

ALMEIDA, H.C.; SILVEIRA, C.B.; ERNANI, P.R.; CAMPOS, M.L. \& ALMEIDA, D. Composição química de um resíduo alcalino da indústria de papel e celulose (DREGS). Química Nova, 30:1669-1672, 2007.

BENDFELDT, E.S.; BURGER, J.A. \& DANIELS, W.L. Quality of amended mine soils after sixteen years. Soil Sci. Soc. Am. J., 65:1736-1744, 2001.

BRADSHAW, A. Restoration of mined lands using natural processes. Ecol. Eng., 8:255-269, 1997.

BRASIL. Ministério da Agricultura. Departamento Nacional da Produção Mineral (DNPM). Perfil analítico do carvão. 2.ed. Porto Alegre, 1987. 140p. (Boletim, 6)

CAMPOS, M.L.; ALMEIDA, J.A. \& SOUZA, L.S. Avaliação de três áreas de solo construído após mineração de carvão a céu aberto em Lauro Müller, Santa Catarina. R. Bras. Ci. Solo, 27:1123-1137, 2003.

CHONG, S.K. \& COWAERT, P.T. Infiltration in reclaimed mined land ameliorated with deep tillage treatments. Soil Till. Res., 44:255-264, 1997.

COMISSÃO DE QUÍMICA E FERTILIDADE DO SOLO CQFSRS/SC. Manual de adubação e de calagem para os Estados do Rio Grande do Sul e Santa Catarina. 10.ed. Porto Alegre, 2004. 400p. 
COSTA, A. Qualidade do solo e desenvolvimento do milho sob diferentes sistemas de manejo na integração lavourapecuária. Lages, Universidade do Estado de Santa Catarina, 2005. 84p. (Tese de Mestrado)

D’ANDRÉA, A.F.; SILVA, M.L.N.; CURI, N. \& FERREIRA, M.M. Atributos de agregação indicadores da qualidade do solo em sistemas de manejo na região dos cerrados no sul do Estado de Goiás. R. Bras. Ci. Solo, 26:1047-1054, 2002.

EMPRESA BRASILEIRA DE PESQUISA AGROPECUÁRIA . EMBRAPA. Centro Nacional de Pesquisa de Solos. Manual de métodos de análise de solo. 2.ed. Rio de Janeiro, 1997. 212p.

EMPRESA BRASILEIRA DE PESQUISA AGROPECUÁRIA EMBRAPA. Centro Nacional de Pesquisa de Solos. Sistema brasileiro de classificação de solos. 2.ed. Brasília, 2006. 306p.

GAIVIZZO, L.H.B.; VIDOR, C.; TEDESCO, M.J. \& BISSANI, C.A. Potencial poluidor de regiões carboníferas. II - Efeitos da recuperação com camadas de solo sobre as plantas e a população microbiana. Ci. Rural, 32:955-961, 2002.

GUEBERT, M.D. \& GARDNER, T.W. Macropore flow on a reclaimed surface mine: Infiltration and hillslope hydrology. Geomorphology, 39:151-169, 2001.

HARMS, T.E. \& CHANASYK, D.S. Plot and small-watershed scale runoff from two reclaimed surface-mined watersheds in Alberta. Hydrol. Proc., 14:1327-1339, 2000.

HILLEL, D. Environmental soil physics. New York, Academic Press, 1998. 770p.

INDORANTE, S.J.; JANSEN, I.J. \& BOAST, C.W. Surface mining and reclamation: Initial changes in soil character. J. Soil Water Conserv., 36:347-351, 1981.

JORDAN, M. \& RODRIGUEZ, E. Effect of solid residues from the cellulose industry on plant growth. J. Plant Nutr. Soil Sci., 167:351-356, 2004.

KÄMPF, N.; SCHNEIDER, P. \& GIASSON, E. Propriedades, pedogênese e classificação de solos construídos em áreas de mineração na Bacia Carbonífera do Baixo Jacuí. R. Bras. Ci. Solo, 21:79-88, 1997.

KEMPER, W.D. \& CHEPIL, W.S. Size distribution of aggregates. In: BLACK, C.A.; EVANS, D.D.; WHITE, J.L.; ENSMINGER, L.E. \& CLARCK, F.E., eds. Methods of soil analysis: Physical and mineralogical methods. Madison, American Society of Agronomy, 1965. p.499510.

MAÇANEIRO, K.C. Efeito da calagem e da adubação orgânica no estabelecimento de gramíneas em área de solo reconstruído após mineração de carvão a céu aberto em Lauro Müller, SC. Lages, Universidade do Estado de Santa Catarina, 2001. 57p. (Tese de Mestrado)

McSWEENEY, K. \& JANSEN, I.J. Soil structure and associated rooting behavior in minesoils. Soil Sci. Soc. Am. J., 48:607 612, 1984.
MELLONI, R.; SIQUEIRA, J.O. \& MOREIRA, F.M.S. Fungos micorrízicos arbusculares em solos de área de mineração de bauxita em reabilitação. Pesq. Agropec. Bras., 38:267276, 2003.

MILLER, W.P.; FRENKEL, H. \& NEWMAN, K.D. Flocculation concentration and sodium calcium exchange of kaolinitic soil clays. Soil. Sci. Soc. Am. J., 54:346-351, 1990.

MOREIRA, P.R. Manejo do solo e recomposição da vegetação com vistas a recuperação de áreas degradadas pela extração de bauxita, Poços de Caldas, MG. Rio Claro, Universidade Estadual Paulista Julio de Mesquita Filho, 2004. 139p. (Tese de Doutorado)

PAULETTO, E.A.; LEITZKE, V.W.; PINTO, L.F.S.; NUNES, M.C.D.; HARTWIG, M.P.; SANTOS, E.L.; WESTPHAL, M.R. \& TEIXEIRA, C.F.A. Água disponível de solos construídos na área de mineração de carvão de Candiotal RS. In: CONGRESSO BRASILEIRO DE CIÊNCIA DO SOLO, 29., Ribeirão Preto, 2003. Anais. Ribeirão Preto, Universidade Estadual Paulista, 2003. CD-ROM.

REICHERT, J.M.; REINERT, D.J. \& BRAIDA, J.A. Qualidade dos solos e sustentabilidade de sistemas agrícolas. Ci. Amb., 27:29-48, 2003.

SANTA CATARINA. Gabinete de Planejamento e Coordenação Geral. Sub-chefia de Estatística, Geografia e Informática. Atlas escolar de Santa Catarina. Rio de Janeiro, Aerofoto Cruzeiro, 1991. 96p.

SHUKLA, M.K.; LAL, R.; UNDERWOOD, J. \& EBINGER, M. Physical and hydrological characteristics of reclaimed mine soils in Southeastern Ohio. Soil Sci. Soc. Am. J., 68:1352$1359,2004$.

SILVA, I.F. \& MIELNICZUK, J. Ação do sistema radicular de plantas na formação e estabilização de agregados do solo. R. Bras. Ci. Solo, 21:113-117, 1997.

SOARES, E.R.; MELLO, J.W.V.; SCHAEFER, C.E.G.R. \& COSTA, L.M. Cinza e carbonato de cálcio na mitigação de drenagem ácida em estéril de mineração. R. Bras. Ci. Solo, 30:171-181, 2006.

SUZUKI, L.E.A.S.; REINERT, D.J.; KAISER, D.R.; KUNZ, M.; PELEGRINI, A.; REICHERT, J.M. \& ALBUQUERQUE, J.A. Teor de argila de solos sob diferentes tempos de agitação horizontal, tempo de contato do dispersante químico e dispersão mecânica. In: REUNIÃO BRASILEIRA DE MANEJO E CONSERVAÇÃO DO SOLO E DA ÁGUA, 15., Santa Maria, 2004. Anais. Santa Maria, Sociedade Brasileira de Ciência do Solo, 2004. CDROM.

TEDESCO, M.J.; GIANELLO, C.; BISSANI, C.A.; BOHNEN, H. \& VOLKWEISS, S.J. Análises de solo, plantas e outros materiais. 2.ed. Porto Alegre, Universidade Federal do Rio Grande do Sul, 1995. 174p.

TORBERT, J.L.; BURGER, J.A. \& DANIELS, W.L. Pine growth variation associated with overburden rock type on a reclaimed surface mine in Virginia. J. Environ. Qual., 19:88-92, 1990. 
WALDEMAR, C.C. \& HERRERA, J. Avaliação do potencial de utilização do "dregs" e do grits como corretivo de acidez e fertilizante na agricultura. In: CONGRESSO ANUAL DE CELULOSE E PAPEL, 19., São Paulo, 1986. Trabalhos técnicos. São Paulo, Associação Brasileira de Celulose e Papel, 1986. p.447-453.
ZAMBRANO, M.; PARODI, V.; GALLARDO, F. \& VIDAL, G. Characterization of "dregs" and "grits" from cellulose paste industry: Study for its application to acid soils. Afinidad, 60:16-25, 2003. 